\title{
Further Results on the Stability Analysis of Singular Systems with Time-Varying Delay: A Delay Decomposition Approach
}

\begin{abstract}
Pin-Lin Liu
Department of Automation, Engineering Institute of Mechatronoptic System, Chienkuo Technology University, Changhua 500, Taiwan

Correspondence should be addressed to Pin-Lin Liu; lpl@ctu.edu.tw

Received 25 August 2012; Accepted 25 September 2012

Academic Editor: Malte Braack

Copyright (C) 2013 Pin-Lin Liu. This is an open access article distributed under the Creative Commons Attribution License, which permits unrestricted use, distribution, and reproduction in any medium, provided the original work is properly cited.

This paper deals with the problem of stability analysis for singular systems with time-varying delay. By developing a delay decomposition approach, information of the delayed plant states can be taken into full consideration, and new delay-dependent sufficient stability criteria are obtained in terms of linear matrix inequalities (LMIs), which can be easily solved by various optimization algorithms. The merits of the proposed results lie in their less conservatism which is realized by choosing different Lyapunov matrices in the decomposed intervals and taking the information of the delayed plant states into full consideration. It is proved that the newly proposed criteria may introduce less conservatism than some existing ones. Meanwhile, the computational complexity of the presented stability criteria is reduced greatly since fewer decision variables are involved. Numerical examples are included to show that the proposed method is effective and can provide less conservative results.
\end{abstract}

\section{Introduction}

Delay phenomena widely exist in many practical engineering systems, such as aircraft, chemical, and process control systems. The study on time delay systems is thus of great significance both in theory and in practice. The time delay is frequently a source of instability and performance deterioration. Therefore, stability analysis and controller synthesis for timedelay system have been one of the most challenging issues [123]. On the other hand, singular time-delay systems, which are also referred to as implicit time-delay systems, descriptor time-delay systems, or generalized differential-difference equations, have strong practical relevance in various engineering systems, including aircraft attitude control, flexible arm control of robots, large-scale electric network control, chemical engineering systems, lossless transmission lines, and so forth (see, e.g., [3-11, 13, 15-21]). For this reason, over the past decades, there has been increasing interest in the stability analysis for singular time-delay systems, and many results have been reported in the literature [6, 9, 15-20].

Recently, some improved delay-dependent stability criteria have been obtained without using different model transformations and bounding techniques for cross terms $[4-6,13$, $16,19]$. However, some slack variables are introduced apart from the matrix variables appearing in Lyapunov-Krasovskii functionals (LKFs), and the results are still conservative, which can be seen by applying these types of criteria to the nominal singular system with a constant time delay and comparing with analytical delay limit for stability. Therefore how one can further improve the existing stability criteria is of great importance to the further study of singular time delay systems. In order to reduce the conservatism, a delay decomposition approach was also proposed in $[9,12,20,21$, $23]$. Both by theory analysis and by numerical examples, it is pointed out that the results obtained by delay decomposition approach are much less conservative than some existing ones and include some as their special cases. Therefore by this approach, very significant steps have been made towards the analytical delay limit for the stability of time-delay systems.

Motivated by the above discussions, we propose new stability criteria for singular systems with time-varying delays. The main aim is to derive a maximum admissible upper bound (MAUB) of the time delay such that the time-delay system is asymptotically stable for any delay size less than the MAUB. Accordingly, the obtained MAUB becomes a key performance index to measure the conservatism of a delaydependent stability condition. The merits of the proposed 
results lie in their less conservatism which is realized by choosing different Lyapunov matrices in the decomposed intervals and taking the information of the delayed plant states into full consideration. The analysis, eventually, culminates into a stability condition in convex linear matrix inequality (LMI) framework and is solved nonconservatively at boundary conditions using standard LMI solvers. Numerical examples are given to illustrate the effectiveness and less conservatism of the proposed method.

\section{Main Result}

Consider the following singular system with a time-varying state delay:

$$
\begin{gathered}
E \dot{x}(t)=A x(t)+B x(t-h(t)), \quad t \geq 0, \\
x(t)=\phi(t), \quad t \in[-h, 0]
\end{gathered}
$$

where $x(t) \in R^{n}$ is the state vector; $A$ and $B$ are constant matrices with appropriate dimensions; the matrix $E \in$ $R^{n \times n}$ may be singular, without loss generality, we suppose rank $E=r \leq n ; \phi(t)$ is a smooth vector-valued initial function; $h(t)$ is a time-varying delay in the state; $h$ is an upper bound on the delay $h(t)$. follows.

We consider two different cases for time varying delays as

Case I. $\quad h(t)$ is a differentiable function, satisfying for all $t \geq$ 0 :

$$
0 \leq h(t) \leq h, \quad \dot{h}(t) \leq h_{d} .
$$

Case II. $h(t)$ is not differentiable or the upper bound of the derivative of $h(t)$ is unknown, and $h(t)$ satisfies

$$
0 \leq h(t) \leq h
$$

where $h$ and $h_{d}$ are some positive constants.

The main objective is to find the range of $h$ and guarantee stability for the singular system with a time-varying state delay (1a) and (1b). Here, definitions and fundamental lemmas are reviewed.

Definition 1 (see [3]). The pair $(E, A)$ is said to be regular if $\operatorname{det}(s E-A)$ is not identically zero.

Definition 2 (see [3]). The pair $(E, A)$ is said to be impulsefree if $\operatorname{deg}(\operatorname{det}(s E-A))=\operatorname{rank} E$.

Definition 3. For a given scalar $\bar{h}>0$, the singular delay system (1a) and (1b) is said to be regular and impulse-free for any constant time delay $h$ satisfying $0 \leq h \leq \bar{h}$, if the pairs $(E, A)$ and $(E, A+B)$ are regular and impulse-free.

Remark 4. The regularity and the absence of impulses of the pair $(E, A)$ ensure the system (1a) and (1b) with time delay $h \neq 0$ to be regular and impulse-free, while the fact that the pair $(E, A+B)$ is regular and impulse-free ensures the system (1a) and (1b) with time delay $h=0$ to be regular and impulsefree.

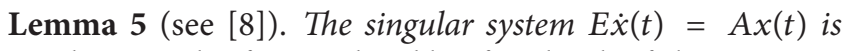
regular, impulse free, and stable, if and only if there exists a matrix $P$ such that

$$
\begin{gathered}
P^{T} E=E^{T} P \geq 0, \\
A^{T} P+P A<0 .
\end{gathered}
$$

Lemma 6 (see [11]). For any positive semidefinite matrices,

$$
X=\left[\begin{array}{lll}
X_{11} & X_{12} & X_{13} \\
X_{12}^{T} & X_{22} & X_{23} \\
X_{13}^{T} & X_{23}^{T} & X_{33}
\end{array}\right] \geq 0,
$$

the following integral inequality holds

$$
\begin{aligned}
-\int_{t-h(t)}^{t} & \dot{x}^{T}(s) X_{33} \dot{x}(s) d s \\
\leq & \int_{t-h(t)}^{t}\left[\begin{array}{ll}
x^{T}(t) & x^{T}(t-h(t)) \dot{x}^{T}(s)
\end{array}\right] \\
& \times\left[\begin{array}{ccc}
X_{11} & X_{12} & X_{13} \\
X_{12}^{T} & X_{22} & X_{23} \\
X_{13}^{T} & X_{23}^{T} & 0
\end{array}\right]\left[\begin{array}{c}
x(t) \\
x(t-h(t)) \\
\dot{x}(s)
\end{array}\right] d s .
\end{aligned}
$$

Secondary, we introduce the following Schur complement which is essential in the proofs of our results.

Lemma 7 (see [2]). The following matrix inequality:

$$
\left[\begin{array}{ll}
Q(x) & S(x) \\
S^{T}(x) & R(x)
\end{array}\right]<0,
$$

$$
Q(x)=Q^{T}(x), R(x)=R^{T}(x) S(x) x,
$$

$$
\begin{gathered}
R(x)<0, \\
Q(x)<0, \\
Q(x)-S(x) R^{-1}(x) S^{T}(x)<0 .
\end{gathered}
$$

This paper finds new stability criteria less conservative than the existing results.

For the system (1a), (1b), and (2), we give stability condition by using a delay decomposition approach as follows.

Theorem 8. In Case I, if $0 \leq h(t) \leq \alpha h$, for given three scalars $h, \alpha$, and $h_{d}$, then, for any delay $h(t)$ satisfy $0 \leq h(t) \leq h$, $\dot{h}(t) \leq h_{d}$, and $0<\alpha<1$, the system described by (1a) and (1b) with (2) is asymptotically stable if there exist matrices 
$P=P^{T}>0, Q_{i}=Q_{i}^{T}>0, R_{i}=R_{i}^{T}>0,(i=1,2,3)$, matrix $S$ of appropriate dimensionsand positive semidefinite matrices: $X=\left[\begin{array}{lll}X_{11} & X_{12} & X_{13} \\ X_{12}^{T} & X_{22} & X_{23} \\ X_{13}^{T} & X_{23}^{T} & X_{33}\end{array}\right] \geq 0, Y=\left[\begin{array}{lll}Y_{11} & Y_{12} & Y_{13} \\ Y_{12}^{T} & Y_{22} & Y_{23} \\ Y_{13}^{T} & Y_{23}^{T} & Y_{33}\end{array}\right] \geq 0, Z=$ $\left[\begin{array}{lll}Z_{11} & Z_{12} & Z_{13} \\ Z_{12}^{T} & Z_{22} & Z_{23} \\ Z_{13}^{T} & Z_{23}^{T} & Z_{33}\end{array}\right] \geq 0$ such that

$$
\begin{gathered}
P^{T} E=E^{T} P \geq 0, \\
\Omega=\left[\begin{array}{ccccc}
\Omega_{11} & \Omega_{12} & 0 & 0 & \Omega_{15} \\
\Omega_{12}^{T} & \Omega_{22} & \Omega_{23} & 0 & \Omega_{25} \\
0 & \Omega_{23}^{T} & \Omega_{33} & \Omega_{34} & 0 \\
0 & 0 & \Omega_{34}^{T} & \Omega_{44} & 0 \\
\Omega_{15}^{T} & \Omega_{25}^{T} & 0 & 0 & \Omega_{55}
\end{array}\right]<0, \\
E^{T}\left(R_{1}-X_{33}\right) E \geq 0, \quad E^{T}\left(R_{2}-Y_{33}\right) E \geq 0, \\
E^{T}\left[R_{1}+\left(1-h_{d}\right) R_{3}-Z_{33}\right] E \geq 0,
\end{gathered}
$$

where $U \in R^{n \times(n-r)}$ is any matrix satisfying $E^{T} U=0$ and

$$
\begin{aligned}
& \Omega_{11}= A^{T} P+P A+Q_{1}+Q_{3}+A^{T} U S^{T}+S U^{T} A \\
&+E^{T}\left(\alpha h Z_{11}+Z_{13}+Z_{13}^{T}\right) E, \\
& \Omega_{12}= P B+S U^{T} B+E^{T}\left(\alpha h Z_{12}-Z_{13}+Z_{23}^{T}\right) E, \\
& \Omega_{15}= A^{T}\left[\alpha h R_{1}+(1-\alpha) h R_{2}+\alpha h R_{3}\right], \\
& \Omega_{22}=-\left(1-h_{d}\right) Q_{3}+E^{T}\left(\alpha h X_{11}+X_{13}+X_{13}^{T}\right. \\
&\left.\quad+\alpha h Z_{22}-Z_{23}-Z_{23}^{T}\right) E, \\
& \Omega_{23}=E^{T}\left(\alpha h X_{12}-X_{13}+X_{23}^{T}\right) E, \\
& \Omega_{25}=B^{T}\left[\alpha h R_{1}+(1-\alpha) h R_{2}+\alpha h R_{3}\right], \\
& \Omega_{33}=Q_{2}-Q_{1}+E^{T}\left[\alpha h X_{22}-X_{23}-X_{23}^{T}\right. \\
&\left.\quad+(1-\alpha) h Y_{11}+Y_{13}+Y_{13}^{T}\right] E, \\
& \Omega_{34}=E^{T}\left[(1-\alpha) h Y_{12}-Y_{13}+Y_{23}^{T}\right] E, \\
& \Omega_{44}=-Q_{2}+E^{T}\left[(1-\alpha) h Y_{22}-Y_{23}-Y_{23}^{T}\right] E, \\
& \Omega_{55}=-\left[\alpha h R_{1}+(1-\alpha) h R_{2}+\alpha h R_{3}\right] .
\end{aligned}
$$

Proof. In Case I, a Lyapunov functional can be constructed as follows:

$$
V\left(x_{t}\right)=V_{1}\left(x_{t}\right)+V_{2}\left(x_{t}\right)+V_{3}\left(x_{t}\right),
$$

where

$$
\begin{aligned}
V_{1}\left(x_{t}\right)= & x^{T}(t) P E x(t), \\
V_{2}\left(x_{t}\right)= & \int_{t-\alpha h}^{t} x^{T}(s) Q_{1} x(s) d s \\
& +\int_{t-h}^{t-\alpha h} x^{T}(s) Q_{2} x(s) d s \\
& +\int_{t-h(t)}^{t} x^{T}(s) Q_{3} x(s) d s, \\
V_{3}\left(x_{t}\right)= & \int_{-\alpha h}^{0} \int_{t+\theta}^{t} \dot{x}^{T}(s) E^{T} R_{1} E \dot{x}(s) d s d \theta \\
& +\int_{-h}^{-\alpha h} \int_{t+\theta}^{t} \dot{x}^{T}(s) E^{T} R_{2} E \dot{x}(s) d s d \theta \\
& +\int_{-h(t)}^{0} \int_{t+\theta}^{t} \dot{x}^{T}(s) E^{T} R_{3} E \dot{x}(s) d s d \theta .
\end{aligned}
$$

Taking time derivative $V\left(x_{t}\right)$ for $t \in[0, \infty)$ along the trajectory (1a) and ( $1 \mathrm{~b}$ ) yields

$$
\dot{V}\left(x_{t}\right)=\dot{V}_{1}\left(x_{t}\right)+\dot{V}_{2}\left(x_{t}\right)+\dot{V}_{3}\left(x_{t}\right),
$$

where

$$
\begin{aligned}
\dot{V}_{1}\left(x_{t}\right)= & \dot{x}^{T}(t) E^{T} P x(t)+x^{T}(t) P E \dot{x}(t) \\
= & x^{T}(t)\left(A^{T} P+P A\right) x(t)+x^{T}(t) P B x(t-h(t)) \\
& +x^{T}(t-h(t)) B^{T} P x(t), \\
\dot{V}_{2}\left(x_{t}\right)= & x^{T}(t)\left(Q_{1}+Q_{3}\right) x(t) \\
& -x^{T}(t-h(t))(1-\dot{h}(t)) Q_{3} x(t-h(t)) \\
& +x^{T}(t-\alpha h)\left(Q_{2}-Q_{1}\right) x(t-\alpha h) \\
& -x^{T}(t-h) Q_{2} x(t-h) \\
\leq & x^{T}(t)\left(Q_{1}+Q_{3}\right) x(t) \\
& -x^{T}(t-h(t))\left(1-h_{d}\right) Q_{3} x(t-h(t)) \\
& +x^{T}(t-\alpha h)\left(Q_{2}-Q_{1}\right) x(t-\alpha h) \\
& -x^{T}(t-h) Q_{2} x(t-h), \\
\dot{V}_{3}\left(x_{t}\right)= & \dot{x}^{T}(t) E^{T}\left[\alpha h R_{1}+(1-\alpha) h R_{2}+h(t) R_{3}\right] E \dot{x}(t) \\
& -\int_{t-\alpha h}^{t} \dot{x}^{T}(s) E^{T} R_{1} E \dot{x}(s) d s
\end{aligned}
$$




$$
\begin{aligned}
& -\int_{t-h}^{t-\alpha h} \dot{x}^{T}(s) E^{T} R_{2} E \dot{x}(s) d s \\
& -(1-\dot{h}(t)) \int_{t-h(t)}^{t} \dot{x}^{T}(s) E^{T} R_{3} E \dot{x}(s) d s \\
\leq & \dot{x}^{T}(t) E^{T}\left[\alpha h R_{1}+(1-\alpha) h R_{2}+\alpha h R_{3}\right] E \dot{x}(t) \\
& -\int_{t-\alpha h}^{t} \dot{x}^{T}(s) E^{T} R_{1} E \dot{x}(s) d s \\
& -\int_{t-h}^{t-\alpha h} \dot{x}^{T}(s) E^{T} R_{2} E \dot{x}(s) d s \\
& -\left(1-h_{d}\right) \int_{t-h(t)}^{t} \dot{x}^{T}(s) E^{T} R_{3} E \dot{x}(s) d s .
\end{aligned}
$$

Now, we estimate the upper bound of the last three terms in inequality (13) as

$$
\begin{aligned}
& -\int_{t-\alpha h}^{t} \dot{x}^{T}(s) E^{T} R_{1} E \dot{x}(s) d s \\
& -\int_{t-h}^{t-\alpha h} \dot{x}^{T}(s) E^{T} R_{2} E \dot{x}(s) d s \\
& -\left(1-h_{d}\right) \int_{t-h(t)}^{t} \dot{x}^{T}(s) E^{T} R_{3} E \dot{x}(s) d s \\
& =-\int_{t-\alpha h}^{t-h(t)} \dot{x}^{T}(s) E^{T} R_{1} E \dot{x}(s) d s \\
& -\int_{t-h}^{t-\alpha h} \dot{x}^{T}(s) E^{T} R_{2} E \dot{x}(s) d s \\
& -\int_{t-h(t)}^{t} \dot{x}^{T}(s) E^{T}\left(R_{1}+\left(1-h_{d}\right) R_{3}\right) E^{T} \dot{x}(s) d s \\
& =-\int_{t-\alpha h}^{t-h(t)} \dot{x}^{T}(s) E^{T}\left(R_{1}-X_{33}\right) E \dot{x}(s) d s \\
& -\int_{t-h}^{t-\alpha h} \dot{x}^{T}(s) E^{T}\left(R_{2}-Y_{33}\right) E \dot{x}(s) d s \\
& -\int_{t-h(t)}^{t} \dot{x}^{T}(s) E^{T}\left(R_{1}+\left(1-h_{d}\right) R_{3}-Z_{33}\right) E \dot{x}(s) d s \\
& -\int_{t-\alpha h}^{t-h(t)} \dot{x}^{T}(s) E^{T} X_{33} E \dot{x}(s) d s \\
& -\int_{t-h}^{t-\alpha h} \dot{x}^{T}(s) E^{T} Y_{33} E \dot{x}(s) d s \\
& -\int_{t-h(t)}^{t} \dot{x}^{T}(s) E^{T} Z_{33} E \dot{x}(s) d s .
\end{aligned}
$$

From integral inequality [11], noticing that $E^{T}\left(R_{1}-X_{33}\right) E \geq$ $0, E^{T}\left(R_{2}-Y_{33}\right) E \geq 0$, and $E^{T}\left(R_{1}+\left(1-h_{d}\right) R_{3}-Z_{33}\right) E \geq 0$ yields the following:

$$
\begin{aligned}
& -\int_{t-\alpha h}^{t-h(t)} \dot{x}^{T}(s) X_{33} \dot{x}(s) d s \\
& \leq \int_{t-\alpha h}^{t-h(t)}\left[\begin{array}{lll}
x^{T}(t-h(t)) & x^{T}(t-\alpha h) \dot{x}^{T}(s)
\end{array}\right] \\
& \times\left[\begin{array}{ccc}
X_{11} & X_{12} & X_{13} \\
X_{12}^{T} & X_{22} & X_{23} \\
X_{13}^{T} & X_{23}^{T} & 0
\end{array}\right]\left[\begin{array}{c}
x(t-h(t)) \\
x(t-\alpha h) \\
\dot{x}(s)
\end{array}\right] d s \\
& \leq x^{T}(t-h(t))(\alpha h-h(t)) X_{11} x(t-h(t)) \\
& +x^{T}(t-h(t))(\alpha h-h(t)) X_{12} x(t-\alpha h) \\
& +x^{T}(t-h(t)) X_{13} \int_{t-\alpha h}^{t-h(t)} \dot{x}(s) d s \\
& +x^{T}(t-\alpha h)(\alpha h-h(t)) X_{12}^{T} x(t-h(t)) \\
& +x^{T}(t-\alpha h)(\alpha h-h(t)) X_{22} x(t-\alpha h) \\
& +x^{T}(t-\alpha h) X_{23} \int_{t-\alpha h}^{t-h(t)} \dot{x}(s) d s \\
& +\int_{t-\alpha h}^{t-h(t)} \dot{x}^{T}(s) d s X_{13}^{T} x(t-h(t)) \\
& +\int_{t-\alpha h}^{t-h(t)} \dot{x}^{T}(s) d s X_{23}^{T} x(t-\alpha h) \\
& \leq x^{T}(t-h(t)) \alpha h X_{11} x(t-h(t)) \\
& +x^{T}(t-h(t)) \alpha h X_{12} x(t-\alpha h) \\
& +x^{T}(t-h(t)) X_{13} \int_{t-\alpha h}^{t-h(t)} \dot{x}(s) d s \\
& +x^{T}(t-\alpha h) \alpha h X_{12}^{T} x(t-h(t)) \\
& +x^{T}(t-\alpha h) \alpha h X_{22} x(t-\alpha h) \\
& +x^{T}(t-\alpha h) X_{23} \int_{t-\alpha h}^{t-h(t)} \dot{x}(s) d s \\
& +\int_{t-\alpha h}^{t-h(t)} \dot{x}^{T}(s) d s X_{13}^{T} x(t-h(t)) \\
& +\int_{t-\alpha h}^{t-h(t)} \dot{x}^{T}(s) d s X_{23}^{T} x(t-\alpha h) \\
& =x^{T}(t-h(t))\left[\alpha h X_{11}+X_{13}^{T}+X_{13}\right] x(t-h(t)) \\
& +x^{T}(t-h(t))\left[\alpha h X_{12}-X_{13}+X_{23}^{T}\right] x(t-\alpha h) \\
& +x^{T}(t-\alpha h)\left[\alpha h X_{12}^{T}-X_{13}^{T}+X_{23}\right] x(t-h(t)) \\
& +x^{T}(t-\alpha h)\left[\alpha h X_{22}-X_{23}-X_{23}^{T}\right] x(t-\alpha h) \text {. }
\end{aligned}
$$


Similarly, we obtain

$$
\begin{aligned}
-\int_{t-h}^{t-\alpha h} & \dot{x}^{T}(s) Y_{33} \dot{x}(s) d s \\
\leq & x^{T}(t-\alpha h)\left[(1-\alpha) h Y_{11}+Y_{13}^{T}+Y_{13}\right] x(t-\alpha h) \\
& +x^{T}(t-\alpha h)\left[(1-\alpha) h Y_{12}-Y_{13}+Y_{23}^{T}\right] x(t-h) \\
& +x^{T}(t-h)\left[(1-\alpha) h Y_{12}^{T}-Y_{13}^{T}+Y_{23}\right] x(t-\alpha h) \\
& +x^{T}(t-h)\left[(1-\alpha) h Y_{22}-Y_{23}-Y_{23}^{T}\right] x(t-h) \\
-\int_{t-h(t)}^{t} & \dot{x}^{T}(s) Z_{33} \dot{x}(s) d s \\
\leq & x^{T}(t)\left[h(t) Z_{11}+Z_{13}^{T}+Z_{13}\right] x(t) \\
& +x^{T}(t)\left[h(t) Z_{12}-Z_{13}+Z_{23}^{T}\right] x(t-h(t)) \\
& +x^{T}(t-h(t))\left[h(t) h Z_{12}^{T}-Z_{13}^{T}+Z_{23}\right] x(t) \\
& +x^{T}(t-h(t))\left[h(t) Z_{22}-Z_{23}-Z_{23}^{T}\right] x(t-h(t)) \\
\leq & x^{T}(t)\left[\alpha h Z_{11}+Z_{13}^{T}+Z_{13}\right] x(t) \\
& +x^{T}(t)\left[\alpha h Z_{12}-Z_{13}+Z_{23}^{T}\right] x(t-h(t)) \\
& +x^{T}(t-h(t))\left[\alpha h Z_{12}^{T}-Z_{13}^{T}+Z_{23}\right] x(t) \\
& +x^{T}(t-h(t))\left[\alpha h Z_{22}-Z_{23}-Z_{23}^{T}\right] x(t-h(t))
\end{aligned}
$$

The operator for term $\dot{x}^{T}(t) E^{T}\left[\alpha h R_{1}+(1-\alpha) h R_{2}+\right.$ $\left.\alpha h R_{3}\right] E \dot{x}(t)$ is as follows:

$$
\begin{aligned}
\dot{x}^{T}(t) E^{T} & {\left[\alpha h R_{1}+(1-\alpha) h R_{2}+\alpha h R_{3}\right] E \dot{x}(t) } \\
= & {[A x(t)+B x(t-h(t))]^{T} } \\
& \times\left[\alpha h R_{1}+(1-\alpha) h R_{2}+\alpha h R_{3}\right] \\
& \times[A x(t)+B x(t-h(t))] \\
= & x^{T}(t) A^{T}\left[\alpha h R_{1}+(1-\alpha) h R_{2}+\alpha h R_{3}\right] A x(t) \\
& +x^{T}(t) A^{T}\left[\alpha h R_{1}+(1-\alpha) h R_{2}+\alpha h R_{3}\right] \\
& \times B x(t-h(t))+x^{T}(t-h(t)) B^{T} \\
& \times\left[\alpha h R_{1}+(1-\alpha) h R_{2}+\alpha h R_{3}\right] \\
& \times A x(t)+x^{T}(t-h(t)) B^{T} \\
& \times\left[\alpha h R_{1}+(1-\alpha) h R_{2}+\alpha h R_{3}\right] B x(t-h(t)) .
\end{aligned}
$$

Furthermore, noting $E^{T} U=0$ deduce

$$
0=2 \dot{x}^{T}(t) E^{T} U S^{T} x(t)
$$

Combining (11)-(18) yields the following:

$$
\begin{aligned}
\dot{V}\left(x_{t}\right) \leq & \xi^{T}(t) \Xi \xi(t) \\
& -\int_{t-\alpha h}^{t-h(t)} \dot{x}^{T}(s) E^{T}\left(R_{1}-X_{33}\right) E \dot{x}(s) d s \\
& -\int_{t-h}^{t-\alpha h} \dot{x}^{T}(s) E^{T}\left(R_{2}-Y_{33}\right) E \dot{x}(s) d s \\
& -\int_{t-h(t)}^{t} \dot{x}^{T}(s) E^{T}\left(R_{1}+\left(1-h_{d}\right) R_{3}-Z_{33}\right) \\
& \times E \dot{x}(s) d s,
\end{aligned}
$$

where

$$
\begin{aligned}
& \xi^{T}(t)=\left[\begin{array}{llll}
x^{T}(t) & x^{T}(t-h(t)) & x^{T}(t-\alpha h) & x^{T}(t-h)
\end{array}\right], \\
& \Xi=\left[\begin{array}{cccc}
\Xi_{11} & \Xi_{12} & 0 & 0 \\
\Xi_{12}^{T} & \Xi_{22} & \Xi_{23} & 0 \\
0 & \Xi_{23}^{T} & \Xi_{33} & \Xi_{34} \\
0 & 0 & \Xi_{34}^{T} & \Xi_{44}
\end{array}\right],
\end{aligned}
$$

with

$$
\begin{aligned}
\Xi_{11}= & A^{T} P+P A+Q_{1}+Q_{3}+A^{T} U S^{T} \\
& +S U^{T} A+E^{T}\left(\alpha h Z_{11}+Z_{13}+Z_{13}^{T}\right) E \\
& +A^{T}\left[\alpha h R_{1}+(1-\alpha) h R_{2}+\alpha h R_{3}\right] A, \\
\Xi_{12}= & P B+S U^{T} B+E^{T}\left(\alpha h Z_{12}-Z_{13}+Z_{23}^{T}\right) E \\
& +A^{T}\left[\alpha h R_{1}+(1-\alpha) h R_{2}+\alpha h R_{3}\right] B, \\
\Xi_{22}=- & \left(1-h_{d}\right) Q_{3} \\
& +E^{T}\left(h \alpha X_{11}+X_{13}+X_{13}^{T}+\alpha h Z_{22}-Z_{23}-Z_{23}^{T}\right) E \\
& +B^{T}\left[\alpha h R_{1}+(1-\alpha) h R_{2}+\alpha h R_{3}\right] B, \\
\Xi_{23}= & E^{T}\left(\alpha h X_{12}-X_{13}+X_{23}^{T}\right) E, \\
\Xi_{33}= & Q_{2}-Q_{1}+E^{T}\left[\alpha h X_{22}-X_{23}-X_{23}^{T}\right. \\
& \left.+(1-\alpha) h Y_{11}+Y_{13}+Y_{13}^{T}\right] E, \\
\Xi_{34}= & E^{T}\left[(1-\alpha) h Y_{12}-Y_{13}+Y_{23}^{T}\right] E, \\
\Xi_{44}=- & Q_{2}+E^{T}\left[(1-\alpha) h Y_{22}-Y_{23}-Y_{23}^{T}\right] E .
\end{aligned}
$$

For system (1a) and (1b), when $\Xi<0$ if $0 \leq h(t) \leq \alpha h$ the last three terms in (19) are all less than 0 . Thus, by Schur complements [2], we have $\dot{V}\left(x_{t}\right)<0$.

Theorem 9. In Case I, if $\alpha h \leq h(t) \leq h$, for given three scalars $h, \alpha$, and $h_{d}$, then, for any delay $h(t)$ satisfy $0 \leq h(t) \leq$ $h, \dot{h}(t) \leq h_{d}$ and $0<\alpha<1$, the system described by (1a) 
and (1b) with (2) is asymptotically stable if there exist matrices $P=P^{T}>0, Q_{i}=Q_{i}^{T}>0, R_{i}=R_{i}^{T}>0,(i=1,2,3)$, matrix $S$ of appropriate dimensionsand

$$
\begin{aligned}
X & =\left[\begin{array}{lll}
X_{11} & X_{12} & X_{13} \\
X_{12}^{T} & X_{22} & X_{23} \\
X_{13}^{T} & X_{23}^{T} & X_{33}
\end{array}\right] \geq 0, \\
Y & =\left[\begin{array}{lll}
Y_{11} & Y_{12} & Y_{13} \\
Y_{12}^{T} & Y_{22} & Y_{23} \\
Y_{13}^{T} & Y_{23}^{T} & Y_{33}
\end{array}\right] \geq 0, \\
Z & =\left[\begin{array}{lll}
Z_{11} & Z_{12} & Z_{13} \\
Z_{12}^{T} & Z_{22} & Z_{23} \\
Z_{13}^{T} & Z_{23}^{T} & Z_{33}
\end{array}\right] \geq 0
\end{aligned}
$$

such that

$$
\begin{gathered}
P^{T} E=E^{T} P \geq 0, \\
\bar{\Omega}=\left[\begin{array}{ccccc}
\bar{\Omega}_{11} & \bar{\Omega}_{12} & \bar{\Omega}_{13} & 0 & \bar{\Omega}_{15} \\
\bar{\Omega}_{12}^{T} & \bar{\Omega}_{22} & \bar{\Omega}_{23} & \bar{\Omega}_{24} & \bar{\Omega}_{25} \\
\bar{\Omega}_{13}^{T} & \bar{\Omega}_{23}^{T} & \bar{\Omega}_{33} & 0 & 0 \\
0 & \bar{\Omega}_{24}^{T} & 0 & \bar{\Omega}_{44} & 0 \\
\bar{\Omega}_{15}^{T} & \bar{\Omega}_{25}^{T} & 0 & 0 & \bar{\Omega}_{55}
\end{array}\right]<0, \\
E^{T}\left[R_{1}+\left(1-h_{d}\right) R_{3}-X_{33}\right] E \geq 0, \\
E^{T}\left[R_{2}+\left(1-h_{d}\right) R_{3}-Y_{33}\right] E \geq 0, \\
E^{T}\left(R_{2}-Z_{33}\right) E \geq 0,
\end{gathered}
$$

where $U \in R^{n \times(n-r)}$ is any matrix satisfying $E^{T} U=0$ and

$$
\begin{aligned}
\bar{\Omega}_{11}= & A^{T} P+P A+Q_{1}+Q_{3}+A^{T} U S^{T}+S U^{T} A \\
& +E^{T}\left(\alpha h X_{11}+X_{13}+X_{13}^{T}\right) E, \\
\bar{\Omega}_{12}= & P B+S U^{T} B, \\
\bar{\Omega}_{13}= & E^{T}\left(\alpha h X_{12}-X_{13}+X_{23}^{T}\right) E, \\
\bar{\Omega}_{15}= & A^{T}\left[\alpha h R_{1}+(1-\alpha) h R_{2}+h R_{3}\right], \\
\bar{\Omega}_{22}= & -\left(1-h_{d}\right) Q_{3}+E^{T}\left[(1-\alpha) h Y_{22}-Y_{23}-Y_{23}^{T}\right. \\
\quad & \left.+(1-\alpha) h Z_{11}+Z_{13}+Z_{13}^{T}\right] E, \\
\bar{\Omega}_{23}= & E^{T}\left[(1-\alpha) h Y_{12}^{T}-Y_{13}^{T}+Y_{23}\right] E,
\end{aligned}
$$

$$
\begin{aligned}
& \bar{\Omega}_{24}=E^{T}\left[(1-\alpha) h Z_{12}-Z_{13}+Z_{23}^{T}\right] E, \\
& \bar{\Omega}_{25}=B^{T}\left[\alpha h R_{1}+(1-\alpha) h R_{2}+h R_{3}\right] \text {, } \\
& \bar{\Omega}_{33}=Q_{2}-Q_{1}+E^{T}\left[\alpha h X_{22}-X_{23}-X_{23}^{T}\right. \\
& \left.+(1-\alpha) h Y_{11}+Y_{13}+Y_{13}^{T}\right] E, \\
& \bar{\Omega}_{44}=-Q_{2}+E^{T}\left[(1-\alpha) h Z_{22}-Z_{23}-Z_{23}^{T}\right] E \text {, } \\
& \bar{\Omega}_{55}=-\left[\alpha h R_{1}+(1-\alpha) h R_{2}+h R_{3}\right] .
\end{aligned}
$$

Proof. If $\alpha h \leq h(t) \leq h$, it gets

$$
\begin{aligned}
& -\int_{t-\alpha h}^{t} \dot{x}^{T}(s) E^{T} R_{1} E \dot{x}(s) d s \\
& -\int_{t-h}^{t-\alpha h} \dot{x}^{T}(s) E^{T} R_{2} E \dot{x}(s) d s
\end{aligned}
$$$$
-\left(1-h_{d}\right) \int_{t-h(t)}^{t} \dot{x}^{T}(s) E^{T} R_{3} E \dot{x}(s) d s
$$$$
=-\int_{t-\alpha h}^{t} \dot{x}^{T}(s) E^{T}\left[R_{1}+\left(1-h_{d}\right) R_{3}\right] E \dot{x}(s) d s
$$$$
-\int_{t-h(t)}^{t-\alpha h} \dot{x}^{T}(s) E^{T}\left[R_{2}+\left(1-h_{d}\right) R_{3}\right] E \dot{x}(s) d s
$$$$
-\int_{t-h}^{t-h(t)} \dot{x}^{T}(s) E^{T} R_{2} E \dot{x}(s) d s
$$$$
=-\int_{t-\alpha h}^{t} \dot{x}^{T}(s) E^{T}\left[R_{1}+\left(1-h_{d}\right) R_{3}-X_{33}\right] E \dot{x}(s) d s
$$$$
-\int_{t-h(t)}^{t-\alpha h} \dot{x}^{T}(s) E^{T}\left[R_{2}+\left(1-h_{d}\right) R_{3}-Y_{33}\right] E \dot{x}(s) d s
$$$$
-\int_{t-h}^{t-h(t)} \dot{x}^{T}(s) E^{T}\left(R_{2}-Z_{33}\right) E \dot{x}(s) d s
$$$$
-\int_{t-\alpha h}^{t} \dot{x}^{T}(s) E^{T} X_{33} E \dot{x}(s) d s
$$$$
-\int_{t-h(t)}^{t-\alpha h} \dot{x}^{T}(s) E^{T} Y_{33} E \dot{x}(s) d s
$$$$
-\int_{t-h}^{t-h(t)} \dot{x}^{T}(s) E^{T} Z_{33} E \dot{x}(s) d s .
$$

From integral inequality [11], notice that $E^{T}\left[R_{1}+(1-\right.$ $\left.\left.h_{d}\right) R_{3}-X_{33}\right] E \geq 0, E^{T}\left(R_{2}-Z_{33}\right) E \geq 0$, and $E^{T}\left[R_{2}+(1-\right.$ $\left.\left.h_{d}\right) R_{3}-Y_{33}\right] E \geq 0$ yields

$$
\begin{aligned}
-\int_{t-\alpha h}^{t} & \dot{x}^{T}(s) X_{33} \dot{x}(s) d s \\
\leq & x^{T}(t)\left[\alpha h X_{11}+X_{13}^{T}+X_{13}\right] x(t) \\
& +x^{T}(t)\left[\alpha h X_{12}-X_{13}+X_{23}^{T}\right] x(t-\alpha h)
\end{aligned}
$$




$$
\begin{aligned}
& +x^{T}(t-\alpha h)\left[\alpha h X_{12}^{T}-X_{13}^{T}+X_{23}\right] x(t) \\
& +x^{T}(t-\alpha h)\left[\alpha h X_{22}-X_{23}-X_{23}^{T}\right] x(t-\alpha h), \\
& -\int_{t-h(t)}^{t-\alpha h} \dot{x}^{T}(s) Y_{33} \dot{x}(s) d s \\
& \leq x^{T}(t-\alpha h)\left[(1-\alpha) h Y_{11}+Y_{13}^{T}+Y_{13}\right] \\
& \times x(t-\alpha h)+x^{T}(t-\alpha h) \\
& \times\left[(1-\alpha) h Y_{12}-Y_{13}+Y_{23}^{T}\right] x(t-h(t)) \\
& +x^{T}(t-h(t))\left[(1-\alpha) h Y_{12}^{T}-Y_{13}^{T}+Y_{23}\right] \\
& \times x(t-\alpha h)+x^{T}(t-h(t)) \\
& \times\left[(1-\alpha) h Y_{22}-Y_{23}-Y_{23}^{T}\right] x(t-h(t)), \\
& -\int_{t-h}^{t-h(t)} \dot{x}^{T}(s) Z_{33} \dot{x}(s) d s \\
& \leq x^{T}(t-h(t))\left[(1-\alpha) h Z_{11}+Z_{13}^{T}+Z_{13}\right] \\
& \times x(t-h(t))+x^{T}(t-h(t)) \\
& \times\left[(1-\alpha) h Z_{12}-Z_{13}+Z_{23}^{T}\right] x(t-h) \\
& +x^{T}(t-h)\left[(1-\alpha) h Z_{12}^{T}-Z_{13}^{T}+Z_{23}\right] \\
& \times x(t-h(t))+x^{T}(t-h) \\
& \times\left[(1-\alpha) h Z_{22}-Z_{23}-Z_{23}^{T}\right] x(t-h) .
\end{aligned}
$$

Combining (11)-(18) and (26)-(28) yields

$$
\begin{aligned}
\dot{V}\left(x_{t}\right) \leq & \xi^{T}(t) \bar{\Xi} \xi(t) \\
& -\int_{t-\alpha h}^{t} \dot{x}^{T}(s) E^{T}\left[R_{1}+\left(1-h_{d}\right) R_{3}-X_{33}\right] \\
& \times E \dot{x}(s) d s-\int_{t-h(t)}^{t-\alpha h} \dot{x}^{T}(s) E^{T} \\
& \times\left[R_{2}+\left(1-h_{d}\right) R_{3}-Y_{33}\right] E \dot{x}(s) d s \\
& -\int_{t-h}^{t-h(t)} \dot{x}^{T}(s) E^{T}\left(R_{2}-Z_{33}\right) E \dot{x}(s) d s
\end{aligned}
$$

where

$$
\bar{\Xi}=\left[\begin{array}{cccc}
\bar{\Xi}_{11} & \bar{\Xi}_{12} & \bar{\Xi}_{13} & 0 \\
\bar{\Xi}_{12}^{T} & \bar{\Xi}_{22} & \bar{\Xi}_{23} & \bar{\Xi}_{24} \\
\bar{\Xi}_{13}^{T} & \bar{\Xi}_{23}^{T} & \bar{\Xi}_{33} & 0 \\
0 & \bar{\Xi}_{24}^{T} & 0 & \bar{\Xi}_{44}
\end{array}\right],
$$

For system (1a) and (1b), when $\Xi<0$ if $\alpha h \leq h(t) \leq h$; the last three terms in (29) are all less than 0. Thus, by Schur complements [2], we have $\dot{V}\left(x_{t}\right)<0$.

Theorem 10. In Case II, if $0 \leq h(t) \leq \alpha h$, for given two scalars $h$ and $\alpha$, then, for any delay $h(t)$ satisfy $0 \leq h(t) \leq h$ and $0<\alpha<1$, the system described by (1a) and (1b) with (3) is asymptotically stable if there exist matrices $P=P^{T}>0, Q_{i}=$ $Q_{i}^{T}>0, R_{i}=R_{i}^{T}>0,(i=1,2)$, matrix $S$ of appropriate dimensions and positive semidefinite matrices:

$$
\begin{aligned}
& X=\left[\begin{array}{lll}
X_{11} & X_{12} & X_{13} \\
X_{12}^{T} & X_{22} & X_{23} \\
X_{13}^{T} & X_{23}^{T} & X_{33}
\end{array}\right] \geq 0 \\
& Y=\left[\begin{array}{lll}
Y_{11} & Y_{12} & Y_{13} \\
Y_{12}^{T} & Y_{22} & Y_{23} \\
Y_{13}^{T} & Y_{23}^{T} & Y_{33}
\end{array}\right] \geq 0 \\
& Z=\left[\begin{array}{lll}
Z_{11} & Z_{12} & Z_{13} \\
Z_{12}^{T} & Z_{22} & Z_{23} \\
Z_{13}^{T} & Z_{23}^{T} & Z_{33}
\end{array}\right] \geq 0
\end{aligned}
$$

such that

$$
P^{T} E=E^{T} P \geq 0
$$




$$
\begin{gathered}
\Psi=\left[\begin{array}{ccccc}
\Psi_{11} & \Psi_{12} & 0 & 0 & \Psi_{15} \\
\Psi_{12}^{T} & \Psi_{22} & \Psi_{23} & 0 & \Psi_{25} \\
0 & \Psi_{23}^{T} & \Psi_{33} & \Psi_{34} & 0 \\
0 & 0 & \Psi_{34}^{T} & \Psi_{44} & 0 \\
\Psi_{15}^{T} & \Psi_{25}^{T} & 0 & 0 & \Psi_{55}
\end{array}\right]<0, \\
E^{T}\left(R_{1}-X_{33}\right) E \geq 0, \quad E^{T}\left(R_{2}-Y_{33}\right) E \geq 0, \\
E^{T}\left(R_{1}-Z_{33}\right) E \geq 0,
\end{gathered}
$$

where $U \in R^{n \times(n-r)}$ is any matrix satisfying $E^{T} U=0$ and

$$
\begin{aligned}
\Psi_{11}= & A^{T} P+P A+Q_{1}+A^{T} U S^{T} \\
& +S U^{T} A+E^{T}\left(h Z_{11}+Z_{13}+Z_{13}^{T}\right) E, \\
\Psi_{12}= & P B+S U^{T} B+E^{T}\left(h Z_{12}-Z_{13}+Z_{23}^{T}\right) E, \\
\Psi_{15}= & A^{T}\left[\alpha h R_{1}+(1-\alpha) h R_{2}\right], \\
\Psi_{22}= & E^{T}\left(\alpha h X_{11}+X_{13}+X_{13}^{T}+h Z_{22}-Z_{23}-Z_{23}^{T}\right), \\
\Psi_{23}= & E^{T}\left(\alpha h X_{12}-X_{13}+X_{23}^{T}\right) E, \\
\Psi_{25}= & B^{T}\left[\alpha h R_{1}+(1-\alpha) h R_{2}\right], \\
\Psi_{33}= & Q_{2}-Q_{1}+E^{T}\left[\alpha h X_{22}-X_{23}-X_{23}^{T}\right. \\
\Psi_{34}= & \left.E^{T}\left[(1-\alpha) h Y_{11}+Y_{13}+Y_{13}^{T}\right] E, Y_{23}^{T}\right] E, \\
\Psi_{44}= & -Q_{2}+E^{T}\left[(1-\alpha) h Y_{22}-Y_{23}-Y_{23}^{T}\right] E, \\
\Psi_{55}= & -\left[\alpha h R_{1}+(1-\alpha) h R_{2}\right] .
\end{aligned}
$$

Theorem 11. In Case II, if $\alpha h \leq h(t) \leq h$, for given two scalars $h$ and $\alpha$, then, for any delay $h(t)$ satisfy $0 \leq h(t) \leq h$ and $0<\alpha<1$, the system described by (1a) and (1b) with (3) is asymptotically stable if there exist matrices $P=P^{T}>0, Q_{i}=$ $Q_{i}^{T}>0, R_{i}=R_{i}^{T}>0,(i=1,2)$, matrix $S$ of appropriate dimensions and positive semidefinite matrices:

$$
\begin{aligned}
X & =\left[\begin{array}{lll}
X_{11} & X_{12} & X_{13} \\
X_{12}^{T} & X_{22} & X_{23} \\
X_{13}^{T} & X_{23}^{T} & X_{33}
\end{array}\right] \geq 0, \\
Y & =\left[\begin{array}{lll}
Y_{11} & Y_{12} & Y_{13} \\
Y_{12}^{T} & Y_{22} & Y_{23} \\
Y_{13}^{T} & Y_{23}^{T} & Y_{33}
\end{array}\right] \geq 0, \\
Z & =\left[\begin{array}{lll}
Z_{11} & Z_{12} & Z_{13} \\
Z_{12}^{T} & Z_{22} & Z_{23} \\
Z_{13}^{T} & Z_{23}^{T} & Z_{33}
\end{array}\right] \geq 0
\end{aligned}
$$

such that

$$
P^{T} E=E^{T} P \geq 0,
$$

$$
\begin{gathered}
\bar{\Psi}=\left[\begin{array}{ccccc}
\bar{\Psi}_{11} & \bar{\Psi}_{12} & \bar{\Psi}_{13} & 0 & \bar{\Psi}_{15} \\
\bar{\Psi}_{12}^{T} & \bar{\Psi}_{22} & \bar{\Psi}_{23} & \bar{\Psi}_{24} & \bar{\Psi}_{25} \\
\bar{\Psi}_{13}^{T} & \bar{\Psi}_{23}^{T} & \bar{\Psi}_{33} & 0 & 0 \\
0 & \bar{\Psi}_{24}^{T} & 0 & \bar{\Psi}_{44} & 0 \\
\bar{\Psi}_{15}^{T} & \bar{\Psi}_{25}^{T} & 0 & 0 & \bar{\Psi}_{55}
\end{array}\right]<0, \\
E^{T}\left[R_{1}-X_{33}\right] E \geq 0, \\
E^{T}\left(R_{2}-Z_{33}\right) E \geq 0,
\end{gathered}
$$

where $U \in R^{n \times(n-r)}$ is any matrix satisfying $E^{T} U=0$ and

$$
\begin{aligned}
& \Psi_{11}= A^{T} P+P A+Q_{1}+A^{T} U S^{T} \\
&+S U^{T} A+E^{T}\left(\alpha h X_{11}+X_{13}+X_{13}^{T}\right) E, \\
& \Psi_{12}= P B+S U^{T} B, \\
& \bar{\Psi}_{13}= E^{T}\left(\alpha h X_{12}-X_{13}+X_{23}^{T}\right) E, \\
& \bar{\Psi}_{15}= A^{T}\left[\alpha h R_{1}+(1-\alpha) h R_{2}\right], \\
& \Psi_{22}= E^{T}\left[(1-\alpha) h Y_{22}-Y_{23}-Y_{23}^{T}+(1-\alpha) h Z_{11}\right. \\
&\left.\quad+Z_{13}+Z_{13}^{T}\right] E, \\
& \bar{\Psi}_{23}= E^{T}\left[(1-\alpha) h Y_{12}^{T}-Y_{13}^{T}+Y_{23}\right] E, \\
& \bar{\Psi}_{24}= E^{T}\left[(1-\alpha) h Z_{12}-Z_{13}+Z_{23}^{T}\right] E, \\
& \bar{\Psi}_{25}= B^{T}\left[\alpha h R_{1}+(1-\alpha) h R_{2}\right], \\
& \bar{\Psi}_{33}= Q_{2}-Q_{1}+E^{T}\left[\alpha h X_{22}-X_{23}-X_{23}^{T}+(1-\alpha) h Y_{11}\right. \\
&\left.\quad+Y_{13}+Y_{13}^{T}\right] E, \\
& \bar{\Psi}_{44}=-Q_{2}+E^{T}\left[(1-\alpha) h Z_{22}-Z_{23}-Z_{23}^{T}\right] E, \\
& \bar{\Psi}_{55}=-\left[\alpha h R_{1}+(1-\alpha) h R_{2}\right] .
\end{aligned}
$$

In Case II, a Lyapunov functional can be chosen as (11) with $Q_{3}=R_{3}=0$. Similar to the above analysis, one can get that $\dot{V}\left(x_{t}\right)<0$ holds if $\Psi<0(\bar{\Psi}<0)$. Thus, the proof is complete.

Remark 12. In the proof of Theorems 8-11, the interval [ $t-$ $h, t]$ is divided into subintervals $[t-h, t-\alpha h]$ and $[t-\alpha h, t]$; information of delayed state $x(t-\alpha h)$ can be taken into account. It is clear that the Lyapunov function defined in Theorems 8-11 is more general than the ones in $[6,9,15-21]$.

Remark 13. In the previous works except $[4-6,13,16,19]$, the time-delay term $h(t)$ was usually estimated as $h$ when estimating the upper bound of some cross term this may lead to increasing conservatism inevitably. In Theorems 8-11, the value of the upper bound of some cross term is estimated more exactly than the previous methods since $h(t)$ is confined 
to the subintervals $0 \leq h(t) \leq \alpha h$ or $\alpha h \leq h(t) \leq h$. So, such decomposition method may lead to reduction of conservatism.

Remark 14 . In the stability problem, maximum admissible upper bound (MAUB) $\bar{h}$ that ensures singular system with a time-varying state delay (1a) and (1b) is stabilizable for any $h$ can be determined by solving the following quasi-convex optimization problem when the other bound of time-varying delay $h$ is known.

$$
\begin{array}{lc}
\text { Maximize } & \bar{h} \\
\text { Subject to } & \text { Theorems 8-11. }
\end{array}
$$

Inequality (37) is a convex optimization problem and can be obtained efficiently using the MATLAB LMI Toolbox.

For seeking an appropriate $\alpha$ satisfying $0<\alpha<1$, such that the upper bound $h$ of delay $h(t)$ subjecting to (7a), (7b), and $(7 \mathrm{c})$ is maximal, we give an algorithm.

Algorithm 15 (maximizing $h>0$ ).

Step 1. For given $h_{d}$, choose an upper bound on $h$ satisfying (7a), (7b), and (7c), then select this upper bound as the initial value $h_{0}$ of $h$.

$\because$ : Step 2. Set appropriate step lengths, $h_{\text {step }}$ and $\alpha_{\text {step }}$, for $h$ and $\alpha$, respectively. Set $k$ as a counter and choose $k=1$. Meanwhile, let $h=h_{0}+h_{\text {step }}$ and initial value $\alpha_{0}$ of $\alpha$ equal to $\alpha_{\text {step }}$.

$\because$ : Step 3. Let $\alpha=k \alpha_{\text {step }}$; if inequality (7a), (7b), and (7c) is feasible, go to Step 4; otherwise, go to step 5.

Step 4. Let $h_{0}=h, \alpha_{0}=\alpha, k=1$ and $h=h_{0}+h_{\text {step }}$ then go to Step 3.

Step 5. Let $k=k+1$. If $k \alpha_{\text {step }}<1$, then go to Step 3; otherwise, stop.

Remark 16. For Algorithm 15, final $h_{0}$ is the desired maximum of the upper bound of delay $h(t)$ satisfying (7a), (7b), and (7c), and $\alpha_{0}$ is the corresponding value of $\alpha$.

Remark 17. Similar to Algorithm 15, we can also find an appropriate scalar $\alpha$, such that the upper bound of delay $0 \leq$ $h(t) \leq \alpha h$ subjecting to (32a), (32b), and (32c) attains the maximum.

Remark 18. Similar to Algorithm 15, an algorithm for seeking appropriate $\alpha$ such that the upper bound of delay $\alpha h \leq h(t) \leq$ $h$ subjecting to (23a), (23b), and (23c) and (35a), (35b), and $(35 \mathrm{c})$ are maximal can be easily obtained.

\section{Illustrative Examples}

To show usefulness of our result, let us consider the following numerical examples.
TABLE 1: Comparison of delay-dependent stability conditions in Example 19.

\begin{tabular}{lcc}
\hline Methods & MAUB $(\bar{h})$ & Number of variables \\
\hline$[15]$ & 1.1547 & 53 \\
{$[17]$} & 1.1547 & 33 \\
{$[6]$} & 1.1547 & 24 \\
{$[16]$} & 1.1547 & 17 \\
{$[19]$} & 1.2011 & 13 \\
{$[9]$} & 1.1898 & 13 \\
{$[18]$} & 1.2052 & 33 \\
{$[20]$} & 1.2060 & 21 \\
Theorem $8(\alpha=0.5)$ & 2.3092 & 11 \\
\hline
\end{tabular}

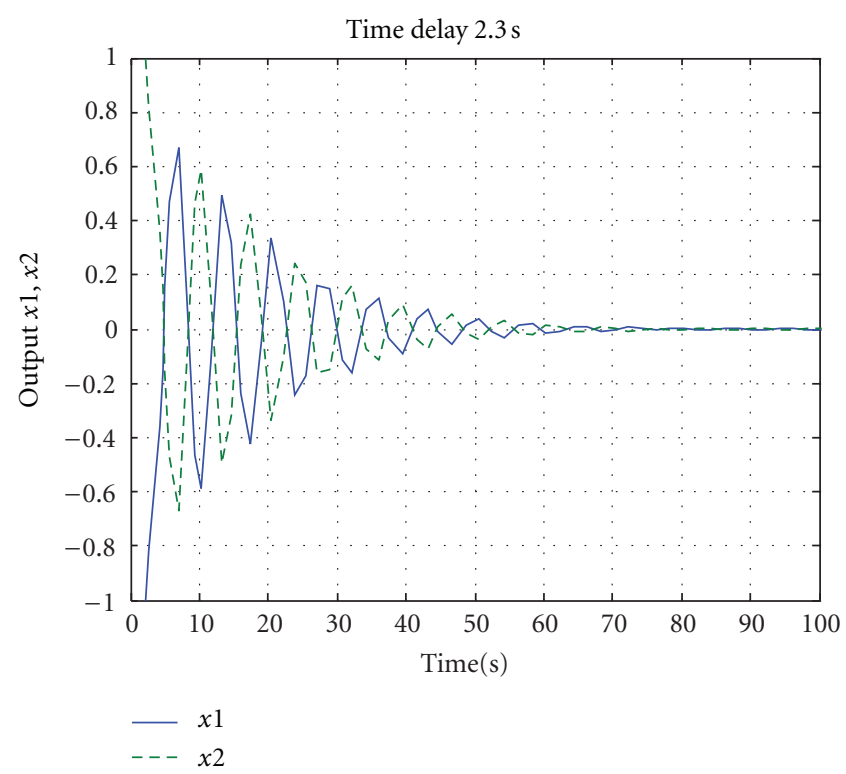

Figure 1: The simulation of the Example 19 for $h=2.3 \mathrm{sec}$.

Example 19. Consider the following time-delay singular systems:

$$
E \dot{x}(t)=A x(t)+B x(t-h(t))
$$

where $E=\left[\begin{array}{ll}1 & 0 \\ 0 & 0\end{array}\right], A=\left[\begin{array}{cc}0.5 & 0 \\ -1 & -1\end{array}\right], B=\left[\begin{array}{cc}-1 & 0 \\ 0 & 0\end{array}\right]$.

Now, our problem is to estimate the maximum admissible upper bound (MAUB) $\bar{h}$ to keep the stability of system (38).

Solution 1. Choosing $U=\left[\begin{array}{ll}1 & 0\end{array}\right]^{T}$ and applying the LMI Toolbox in MATLAB (with accuracy 0.01), this above time delay singular system (38) is asymptotically stable for delay time satisfying $h \leq 2.3092$. Table 1 lists the results compared with $[6,9,15-20]$. It can be seen from Table 1 that the maximum admissible upper bound (MAUB) $\bar{h}$ by using Theorem 8 is the largest with the fewest variables are computed. Figure 1 shows the simulation of the above system (38) for $h=2.3$ with the initial state $\left[\begin{array}{ll}-1 & 1\end{array}\right]^{T}$. 
TABLE 2: Comparison of maximum admissible upper bound (MAUB) $\bar{h}$ in Example 20.

\begin{tabular}{lcccccc}
\hline Methods & {$[6]$} & {$[13]$} & {$[19]$} & {$[4]$} & {$[10]$} & Theorem 8 $(\alpha=0.4)$ \\
\hline $\bar{h}$ & 1.150 & 1.1547 & 1.1547 & 1.2052 & 2.3810 & 2.8863 \\
\hline
\end{tabular}

TABLE 3: Comparison of maximum admissible upper bound (MAUB) $\bar{h}$ in Example 21.

\begin{tabular}{lcccc}
\hline Methods & {$[21]$} & {$[10]$} & {$[11]$} & Theorem $8(\alpha=0.5)$ \\
\hline $\bar{h}$ & 1.274 & 1.281 & 2.3619 & 2.9618 \\
\hline
\end{tabular}

Example 20. Consider the following time delay singular systems:

$$
E \dot{x}(t)=A x(t)+B x(t-h(t))
$$

where $E=\left[\begin{array}{ll}1 & 0 \\ 0 & 0\end{array}\right], A=\left[\begin{array}{cc}0.5 & 0 \\ 0 & -1\end{array}\right], B=\left[\begin{array}{cc}-1 & 1 \\ 0 & 0.5\end{array}\right]$.

Now, our problem is to estimate the maximum admissible upper bound (MAUB) $\bar{h}$ to keep the stability of system (39).

Solution 2. Choosing $\alpha=0.4, U=\left[\begin{array}{ll}1 & 0\end{array}\right]^{T}$ and applying the LMI Toolbox in MATLAB (with accuracy 0.01), this above time delay singular system (39) is asymptotically stable for delay time satisfying $h \leq 2.8863$. The results for stability conditions in different methods are compared in Table 2. It can be shown that the delay-dependent stability condition in this paper is the best performance.

Example 21. Consider the following time delay singular systems:

$$
E \dot{x}(t)=A x(t)+B x(t-h),
$$

where $E=\left[\begin{array}{lll}1 & 0 & 0 \\ 0 & 1 & 0 \\ 0 & 0 & 0\end{array}\right], A=\left[\begin{array}{ccc}-2 & 0 & 0 \\ 0 & -0.5 & 0 \\ 0 & 0 & 1\end{array}\right], B=\left[\begin{array}{ccc}-1 & 0 & 1 \\ -1 & -1 & -0.1 \\ -1 & 1 & -0.1\end{array}\right]$.

Now, our problem is to estimate the maximum admissible upper bound (MAUB) $\bar{h}$ to keep the stability of system (40).

Solution 3. Choosing $U=\left[\begin{array}{lll}0 & 0 & 1\end{array}\right]^{T}$ and applying the LMI Toolbox in MATLAB (with accuracy 0.01), this above timedelay singular system (40) is asymptotically stable for delay time satisfying $h \leq 2.9618$. The maximum admissible upper bounds on the time-delay form Theorem 8 are shown in Table 3. From Table 3, it can be seen that the stability results obtained in the paper are less conservative than those in $[10,11,21]$.

\section{Conclusion}

In this paper, a delay decomposition approach has been developed to investigate the stability of singular systems with a time-varying delay. By developing a delay decomposition approach, the information of the delayed plant states can be taken into full consideration, and new delay-dependent sufficient stability criteria are obtained in terms of linear matrix inequalities (LMIs). Our proposed results are with the form of LMI and can be easily solved by LMI's toolbox in the Matlab without tuning any parameters. It is proved that the obtained results are less conservative than some existing ones. Meanwhile, the computational complexity of the new stability criteria is reduced greatly since fewer decision variables are involved. An algorithm of seeking appropriate tuning parameter is also presented. Numerical examples have illustrated the effectiveness of the proposed methods.

\section{References}

[1] M. S. Ali and P. Balasubramaniam, "Exponential stability of time-delay systems with nonlinear uncertainties," International Journal of Computer Mathematics, vol. 87, no. 6, pp. 1363-1373, 2010.

[2] S. Boyd, L. El Ghaoui, E. Feron, and V. Balakrishnan, Linear Matrix Inequalities in System and Control Theory, SIAM, Philadelphia, Pa, USA, 1994.

[3] L. Dai, Singular Control Systems, Springer, New York, NY, USA, 1989.

[4] Y. F. Feng, X. L. Zhu, and Q. L. Zhang, "Delay-dependent stability criteria for singular time-delay systems," Acta Automatica Sinica, vol. 36, no. 3, pp. 433-437, 2010.

[5] E. Fridman, "Stability of linear descriptor systems with delay: a Lyapunov-based approach," Journal of Mathematical Analysis and Applications, vol. 273, no. 1, pp. 24-44, 2002.

[6] E. Fridman and U. Shaked, " $H_{\infty}$ control of linear state-delay descriptor systems: an LMI approach," Linear Algebra and its Applications, vol. 351, no. 1, pp. 271-302, 2002.

[7] G. Huanli and X. Bugong, "Delay-dependent state feedback robust stabilization for uncertain singular time-delay systems," Journal of Systems Engineering and Electronics, vol. 19, no. 4, pp. 758-765, 2008.

[8] F. L. Lewis, "A survey of linear singular systems," Circuits, Systems, and Signal Processing, vol. 5, no. 1, pp. 3-36, 1986.

[9] S. Liang, J. Cheng, and M. Huang, "A delay decomposition approach to stability analysis of singular systems with timevarying delay," in Proceedings of the International Conference on Test and Measurement (ICTM '09), pp. 110-114, December 2009.

[10] L. L. Liu, J. G. Peng, and B. W. Wu, "On parameterized Lyapunov-Krasovskii functional techniques for investigating singular time-delay systems," Applied Mathematics Letters, vol. 24, no. 5, pp. 703-708, 2011.

[11] P. L. Liu, "Further results on the exponential stability criteria for time delay singular systems with delay-dependence," International Journal of Innovative Computing, Information and Control, vol. 8, no. 6, pp. 4015-4024, 2012.

[12] P. L. Liu, "A delay decomposition approach to stability analysis of uncertain systems with time-varying delays," ISA Transactions, vol. 51, pp. 694-701, 2012. 
[13] H. Su, X. Ji, and J. Chu, "Delay-dependent robust control for uncertain singular time-delay systems," Asian Journal of Control, vol. 8, no. 2, pp. 180-189, 2006.

[14] X. Wu, Y. Wang, L. Huang, and Y. Zuo, "Robust stability analysis of delayed Takagi-Sugeno fuzzy Hopfield neural networks with discontinuous activation functions," Cognitive Neurodynamics, vol. 4, no. 4, pp. 347-354, 2010.

[15] Z. Wu and W. Zhou, "Delay-dependent robust $H_{\infty}$ control for uncertain singular time-delay systems," IET Control Theory and Applications, vol. 1, no. 5, pp. 1234-1241, 2007.

[16] S. Xu, J. Lam, and Y. Zou, "An improved characterization of bounded realness for singular delay systems and its applications," International Journal of Robust and Nonlinear Control, vol. 18, no. 3, pp. 263-277, 2008.

[17] D. Yue and Q. L. Han, "A delay-dependent stability criterion of neutral systems and its application to a partial element equivalent circuit model," IEEE Transactions on Circuits and Systems I, vol. 51, no. 12, pp. 685-689, 2004.

[18] X. L. Zhu and G. H. Yang, "New results of stability analysis for singular time-delay systems," in Proceedings of the American Control Conference, pp. 4905-4908, St. Louis, Mo, USA, 2009.

[19] S. Zhu, C. Zhang, Z. Cheng, and J. Feng, "Delay-dependent robust stability criteria for two classes of uncertain singular time-delay systems," IEEE Transactions on Automatic Control, vol. 52, no. 5, pp. 880-885, 2007.

[20] S. Zhu, Z. Li, and C. Zhang, "Delay decomposition approach to delay-dependent stability for singular time-delay systems," IET Control Theory and Applications, vol. 4, no. 11, pp. 2613-2620, 2010.

[21] S. Q. Zhu, Z. L. Cheng, and J. Feng, "Delay-dependent robust stability criterion and robust stabilization for uncertain singular time-delay systems," in Proceeding of American Control Conference, pp. 2839-2844, Portland, Ore, USA, June 2005.

[22] X. L. Zhu and G. H. Yang, "Jensen integral inequality approach to stability analysis of continuous-time systems with timevarying delay," IET Control Theory and Applications, vol. 2, no. 6, pp. 524-534, 2008.

[23] X. L. Zhu and G. H. Yang, "New results of stability analysis for systems with time-varying delay," International Journal of Robust and Nonlinear Control, vol. 20, no. 5, pp. 596-606, 2010. 


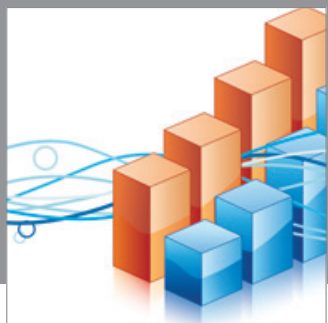

Advances in

Operations Research

mansans

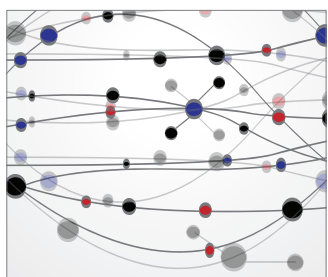

The Scientific World Journal
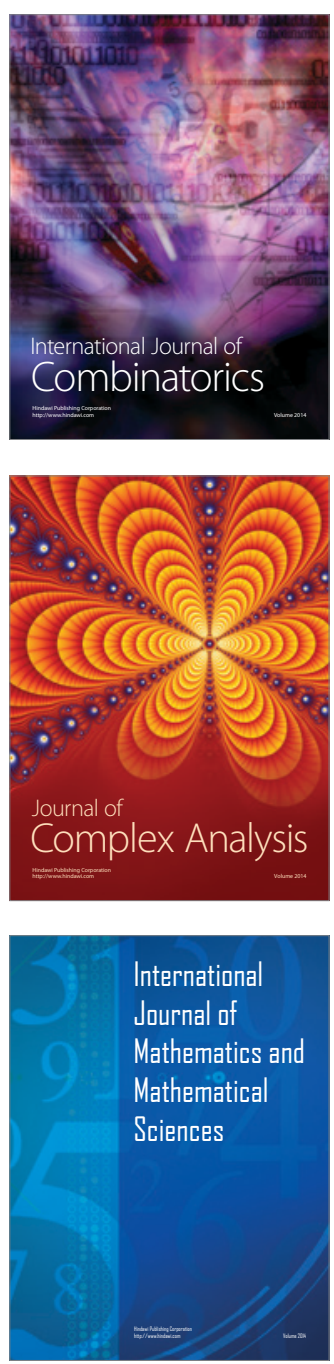
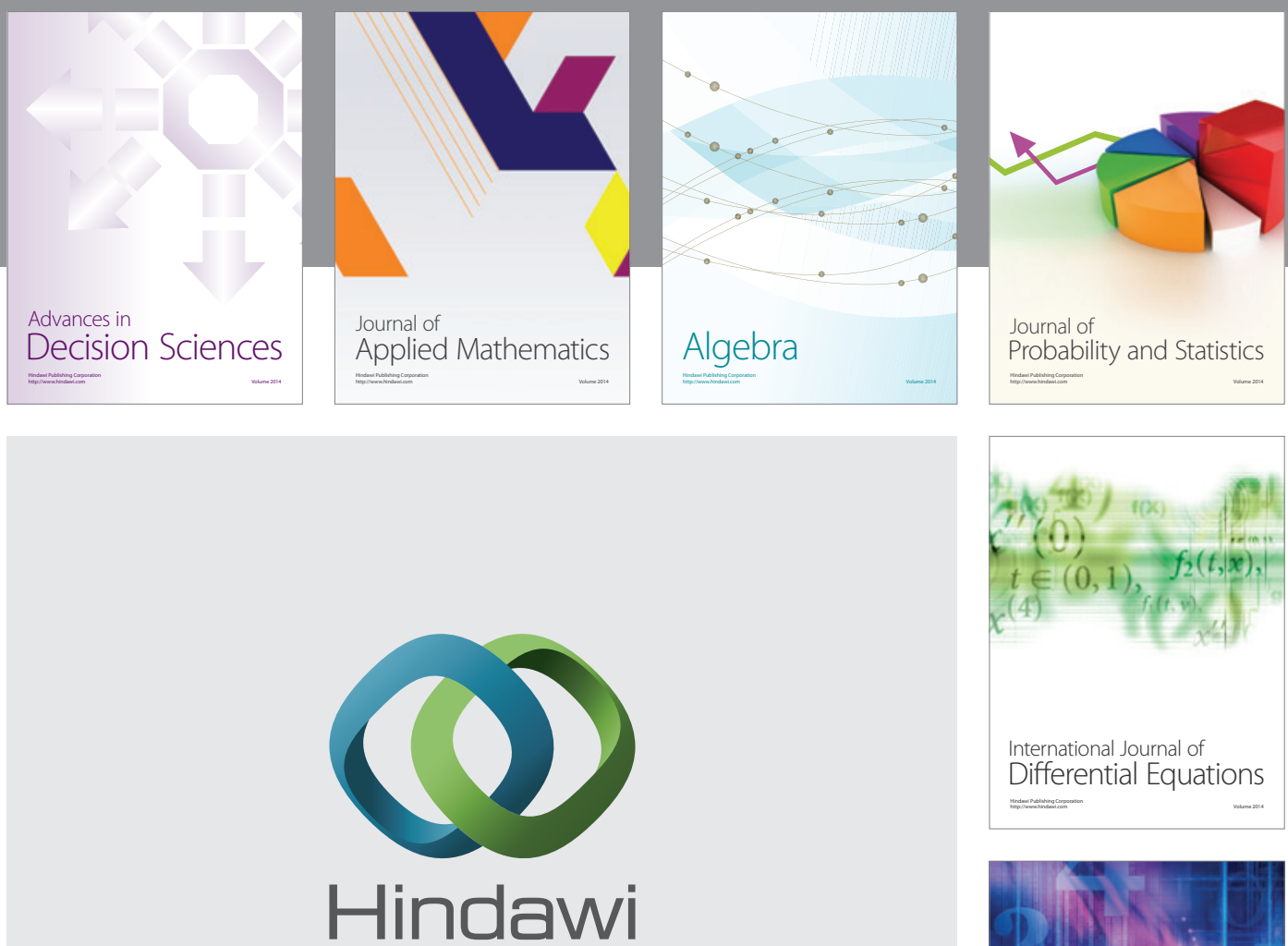

Submit your manuscripts at http://www.hindawi.com
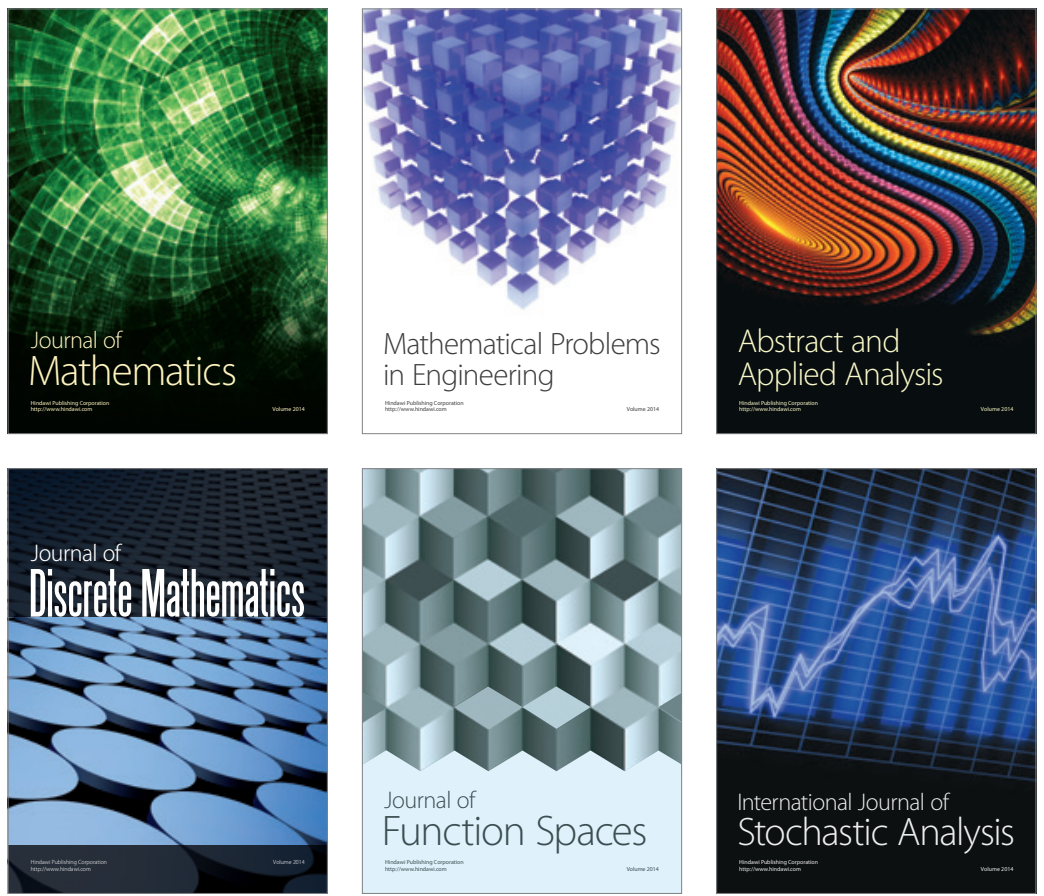

Journal of

Function Spaces

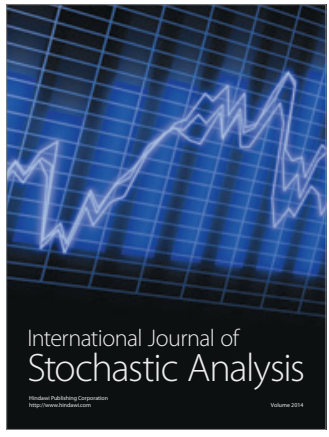

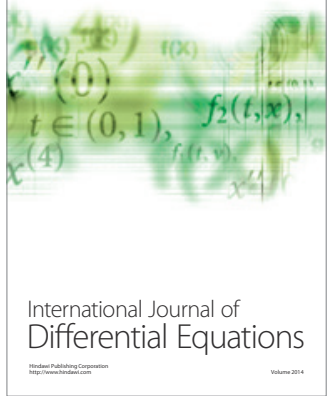
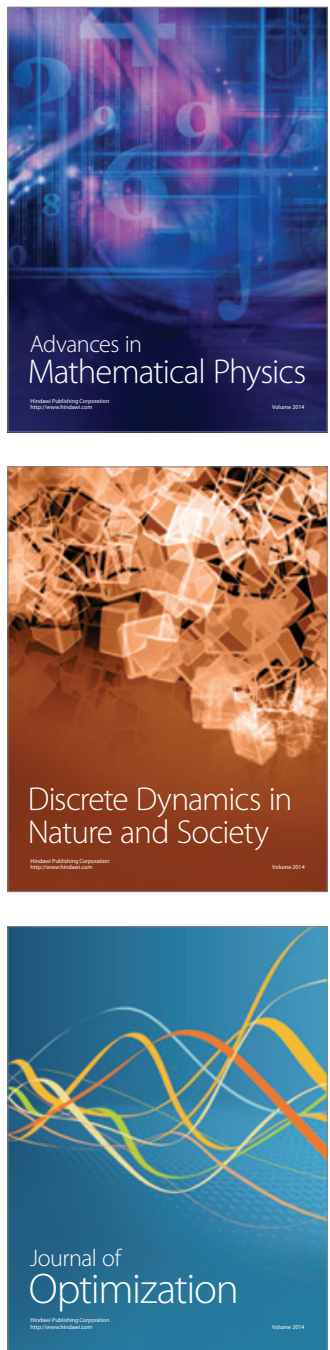